\title{
Harmonic current classification using hybrid FAM-RBF neural network
}

\author{
Shoun Ying Leow ${ }^{1}$, Keem Siah Yap ${ }^{2}$, Shen Yuong Wong ${ }^{3}$ \\ ${ }^{1,2}$ Department of Electronics and Communication f Engineering, Universiti Tenaga Nasional, Malaysia \\ ${ }^{3}$ Department of Electrical and Electronics Engineering, Xiamen University Malaysia, Malaysia
}

\begin{tabular}{|c|c|}
\hline Article Info & ABSTRACT \\
\hline Article history: & In this paper, the type of customers of electricity in Malaysia is classified \\
\hline Received Oct 29, 2019 & A hybrid of Fuzzy Adaptive Resonance Theory with Mapping Algorithm \\
\hline Revised Dec 30, 2019 & (Fuzzy ARTMAP) and Radial Basis Function (RBF) neural network is \\
\hline Accepted Jan 14, 2020 & $\begin{array}{l}\text { developed (namely FAM-RBF), and it is used to classify the harmonic } \\
\text { current into types of consumers. The result of the proposed neural }\end{array}$ \\
\hline Keywords: & $\begin{array}{l}\text { network is discussed, and compared with other neural networks in this paper. } \\
\text { The comparison result shows that the proposed FAM-RBF obtained }\end{array}$ \\
\hline Electrical loads & the best performance result and is a truthful neural network to be used in \\
\hline Fuzzy ARTMAP & \\
\hline Harmonic current & \\
\hline Radial basis function & \\
\hline Type of electricity consumers & $\begin{array}{r}\text { Copyright }(2020 \text { Institute of Advanced Engineering and Science. } \\
\text { All rights reserved. }\end{array}$ \\
\hline \multicolumn{2}{|l|}{ Corresponding Author: } \\
\hline \multicolumn{2}{|c|}{$\begin{array}{l}\text { Shen Yuong Wong, } \\
\text { Department of Electrical and Electronics Engineering, Xiamen University Malaysia, } \\
\text { Jalan Sunsuria, Bandar Sunsuria, } 43900 \text { Sepang, Selangor, Malaysia. } \\
\text { Email: shenyuong.wong@ xmu.edu.my }\end{array}$} \\
\hline
\end{tabular}

\section{INTRODUCTION}

In general, electrical load can be characterized into linear or non-linear electrical loads. Linear load draws the waveform shape that same with the applied voltage. On the other hand, the impedance of nonlinear load changes the applied voltage. The current in a non-linear load will not be sinusoidal even the load is connected to a sinusoidal applied voltage because it is drawn down by the changing impedance in the loads [1]. This non-sinusoidal current contains harmonic current. The harmonic current works together with the impedance of the power distribution system, and generate voltage distortions. These distortions could affect the instruments in the distribution system and the loads connected to it [2].

An aggregate load is formed when numerous of non-linear and linear loads are connected to a low voltage bus of distribution transformer, and consists a wide range of harmonic currents. This connection is known as point of common coupling. The nonlinear loads generate harmonic currents correspondingly with the harmonic current spectrum. Different type of load consumers generate different type of harmonic currents. The major electricity consumption of residential consumers are on electrical home appliances that generate harmonic currents such as computers, ovens and fluorescent lights. However, the energy consumption of commercial consumers are on computers and fluorescent lights [3].

In consequent, harmonic loads can generally be classified to type of load consumers based on character of harmonic currents generated and its total harmonic distortion level. Based on measured statistical data, harmonic currents of aggregate harmonic loads can be connected to load consumers [2]. For a past decade, neural networks (NNs) have been widely applied in power industry and returned a promising results [4]. NN has a strong talent to train and learn from a dataset. The applications of NN in power industry include monitoring active power filter, forecasting electrical loads in short term, analyzing and forecasting 
electrical energy consumption of a building, forecasting the wind power production, and forecasting electric prices [5-8].

In 1987, Adaptive Resonance Theory (ART) was presented firstly by Carpenter and Grossberg. Then, the ART family's size increased rapidly between year 1987 and 1992. Some of the family members are ART-1, ART-2, Fuzzy ART, ART with mapping algorithm (ARTMAP) and Fuzzy ARTMAP (FAM). The rapid growth of ART family is motivated by the remarkable breakthrough of ART in overcome stabilityplasticity dilemma and catastrophic forgetting. ARTMAP is proposed to binary patterns. ARTMAP. Then, Fuzzy ARTMAP (FAM) is extended form ARTMAP, and proposed to binary and analogue patterns. The FAM is capable for online learning. At first, there is no hidden neuron created in FAM. The required hidden neurons of FAM grow based on the training dataset. The FAM is capable to overcome stabilityplasticity dilemma and catastrophic forgetting [9-11]. Several researches are conducted to enhance the performance of FAM and apply FAM in various application [12-16].

On the other hand, the RBF has been catching the attention of researchers recently with the function approximation of its hidden neurons [17]. This research includes enhance the performance of RBF and apply RBF in various industries [7, 18-21]. RBF parks under the group of feedforward NN, which organizes its hidden neurons with function approximation theory. The Gaussian kernel function is a popular choice of the function approximation function. This Gaussian kernel function is computationally and mathematically traceable, and nonzero at any points. In addition, it can be differenced infinitely [22]. The objective of this paper is to extend FAM-RBF for classification of customer type based on harmonic currents in power distribution system. This proposed model uses FAM and RBF to learn the training data samples, and then performance evaluation is obtained based on test samples. The performance of the proposed neural network in harmonic current classification was studied and compare with other popular learning algorithms.

The paper is ordered as below. In Section 2, the learning algorithms of FAM-RBF are presented. After that, Section 3 presents the results and analysis of proposed FAM-RBF neural network for classification of type of customer in power utility. .Lastly, Section V presents a recapitulation of important findings with suggestion for further work.

\section{RESEARCH METHOD}

The FAM NN is made up with a pair of ART modules, and a map field. The ART module is ART-a and ART-b that composed of three neuron layers respectively. The target output must be presented together with the inputs that presented to FAM. Assume the ith training samples presented to the normalization layer of FAM has and input vector with $M$ dimensions, and its target output vector with $L$ dimensions, $\mathbf{X}_{\mathrm{i}} \in \mathbf{R}^{\mathrm{M}}$ and $\mathbf{T}_{\mathrm{i}} \in \mathbf{R}^{\mathrm{L}}$ in vector form, $\left\{\left(\mathbf{X}_{\mathbf{1}}, \mathbf{T}_{\mathbf{1}}\right),\left(\mathbf{X}_{\mathbf{2}}, \mathbf{T}_{2}\right), \ldots,\left(\mathbf{X}_{\mathbf{i}}, \mathbf{T}_{\mathbf{i}}\right)\right\}$. The $F_{0}^{a}$ and $F_{0}^{b}$ are the normalized layer of FAM. The input and its target output vectors are allocated with complement coding, i.e.,

$$
\begin{aligned}
& \boldsymbol{A}_{i}=\left(\mathbf{X}_{i}, \mathbf{X}_{i}^{c}\right)=\left(\mathbf{X}_{i}, 1-\mathbf{X}_{i}\right) \\
& \mathbf{B}_{i}=\left(\mathrm{T}_{i}, \mathrm{~T}_{i}^{c}\right)=\left(\mathrm{T}_{i}, 1-\mathrm{T}_{i}\right)
\end{aligned}
$$

where $\boldsymbol{A}_{i} \in \boldsymbol{R}^{2 M}$ is the complemented inputs in vector form, and $\boldsymbol{B}_{i} \in \boldsymbol{R}^{2 L}$ is its complemented target outputs in vector form. The second layer of FAM is input layer. These layers are labelled as $F_{1}^{a}$ and $F_{1}^{b}$. In this layer, the complemented inputs and its complemented target output which received from normalized layers, are transmitted to next layers, recognition layers. The recognition layers of FAM are labelled as $\boldsymbol{F}_{2}^{a}$ and $\boldsymbol{F}_{2}^{b}$. Both $\boldsymbol{F}_{2}^{a}$ and $\boldsymbol{F}_{2}^{b}$ receive complemented inputs and its complemented target output in vector form respectively from the input layers. In these layers, each pair of inputs and its target outputs are presented simultaneously into the recognition layers of ART-a and ART-b respectively. The number of hidden neurons grows as required during the training process. The FAM practices winner-take-all. The winning neuron is labelled as hidden neuron J. The weight sets of hidden neuron $\mathrm{J}$ which includes hyper-rectangular and centre, is in vector form. The FAM consists Qth hidden neurons. The hyper-rectangular and centres of hidden neurons J in ART-a and ART-b are correspondingly denoted as $\mathbf{w}_{j}^{a}, \mathbf{w}_{j}^{b}, \mathbf{c}_{j}^{a}$, and $\mathbf{c}_{j}^{b}$.

A novel winning hidden neuron $\mathrm{J}$ is created in hidden neurons layer when there is no hidden neurons match with the inputs (i.e., $\mathrm{Q} \leftarrow \mathrm{Q}+1$ ), the centres, $\mathrm{c}$ and weight vectors, $\mathrm{w}$ are fixed correspondingly at 0 and 1. The $q$ is number of hidden neuron $\mathbf{J}$ in $\boldsymbol{F}_{2}^{a}$ layer, has to be set to 0 . The normalized input and its target output vectors of hidden neuron J are presented to ART-a and ART-b respectively and simultaneously during the training period. An activation number is presented to the hidden neuron $\mathrm{J}$ in $\boldsymbol{F}_{2}^{a}$ by the choice function. A choice function, $\boldsymbol{T}_{j}$ which is shown in (2), takes place to select the winning hidden neuron $\mathrm{J}$ selected. The $\boldsymbol{A}_{i}$ is input patterns that propagates from $\boldsymbol{F}_{1}$ layers to $\boldsymbol{F}_{2}$ layers. The $\alpha$ is the choice parameter in the range 
between 0.001 to 10 . The hidden neuron with highest choice function value is selected as the winning hidden neuron.

$$
\boldsymbol{T}_{j}=\frac{\left|\mathbf{A}_{i} \wedge \mathbf{w}_{j}^{a}\right|}{\alpha+\left|\mathbf{w}_{j}^{a}\right|}
$$

Next, a vigilance test which is the essential element of FAM that measure the similarity between the pattern of winning neuron, $\boldsymbol{W}_{J}^{a}, \boldsymbol{A}_{i}$ and threshold, is organised. The comparison is shown in the (3).

$$
\frac{\left|\mathbf{A}_{i} \wedge \mathrm{w}_{J}^{a}\right|}{\left|\mathbf{A}_{i}\right|} \geq \rho_{a}
$$

The vigilance parameter in ART-a, $\rho$ a is a number that range from 0 to 1 . If the winning hidden neuron fails the vigilance test, a winning hidden neuron searching cycle has to be performed continuously until the new winning hidden $\mathbf{J}$ is found. Meanwhile, the same operation takes place in ART-b. After identifying the winning neuron in $F_{2}^{\mathbf{a}}$, the hidden neuron of its target output in $F_{2}^{\text {b }}$ (i.e., ${ }^{\mathbf{w}_{J}^{b}}$ ) is engaged to execute vigilance test in ART-b. The double confirm of new hidden neuron $J$ is the final winning hidden neuron is indeed. The comparison in art-b is shown in (4):

$$
\frac{\left|\mathbf{B}_{i} \wedge \mathbf{w}_{J}^{b}\right|}{\left|\mathbf{B}_{i}\right|} \geq \rho_{b}
$$

The vigilance parameter in art-b, $\rho b$ is a number that range from 0 to 1 . If vigilance test is failed, tracking match process takes place. If there is no existing hidden neurons that fulfil the (4), a new hidden neuron has to be added.

$$
\rho_{a}=\frac{\left|\mathbf{A}_{i} \wedge \mathbf{w}_{J}^{a}\right|}{\left|\mathbf{A}_{i}\right|}+0.00001
$$

The 0.00001 is a constant value added to avoid the re-selection of hidden neuron $\mathrm{J}$ in $F_{2}^{a}$. A different threshold value of $\rho_{a}$ takes place to start a new search cycle in ART-a. When the winning hidden neuron $\mathrm{J}$ is found, its $\mathrm{w}$ and $\mathrm{c}$ in $F_{2}^{a}$ and $F_{2}^{b}$ are restructured as below:

$$
\begin{aligned}
& \mathbf{w}_{J}^{a} \leftarrow \mathbf{A}_{i} \wedge \mathbf{w}_{J}^{a}, \mathbf{w}_{J}^{b} \leftarrow \mathbf{B}_{i} \wedge \mathbf{w}_{J}^{b} \\
& \boldsymbol{q}_{J} \leftarrow \boldsymbol{q}_{J}+1 \\
& c_{J}^{a} \leftarrow\left(1-\frac{1}{q_{J}}\right) c_{J}^{a}+\frac{\mathbf{A}_{i}}{q_{J}}, c_{J}^{b} \leftarrow\left(1-\frac{1}{q_{J}}\right) c_{J}^{b}+\frac{\mathbf{B}_{i}}{q_{J}}
\end{aligned}
$$

The Radial Basis Function (RBF) is firstly introduced in 1991. It is a universal function approximation. A RBF NN contains only three layers, i.e., input, hidden neuron, and output layers. A notable feature of RBF NN is its hidden neurons need an activation function to activate them. The hidden neurons practice non-linear and local mapping. In this paper, Gaussian function is selected. The absolute value of this function decreases continuously approaches zero in all dimensions when the inputs are away from its center [23]. A major challenge in the design of Gaussian function is the selection of its centers. Assume a RBF NN has P input neurons, Q hidden neurons and R output neurons. The output of the RBF NN, $y_{r}$ is computed in (9). 
$y_{r}=\sum_{j=1}^{J} \boldsymbol{\beta}_{j r} G_{j}\left(\mathbf{X}, c_{j}^{a}, \sigma\right)=\sum_{j=1}^{Q_{a}} \beta_{j r} \exp \left(-\frac{\left\|\boldsymbol{X}_{p}-\mathbf{c}_{j}^{a}\right\|^{2}}{2 \sigma^{2}}\right)$

The $r=1,2, \ldots, \mathrm{R}$ is the output neurons of RBF NN. However, $\mathbf{G}_{j}, \beta_{j r}$ and $c_{j}$ are activation function, output weight and center of the hidden neurons respectively. The parameter of RBF NN, $\sigma$ is the width of the Gaussian function, which is an adjustable parameter, has be manually determined before its training. The Gaussian function, $\mathbf{G}_{j}$ is shown in (10):

$$
\mathbf{G}_{j}\left(\mathbf{X}, c_{j}^{a}, \sigma\right)=\exp \left(-\frac{\left\|\boldsymbol{X}_{p}-\mathbf{c}_{j}^{a}\right\|^{2}}{2 \sigma^{2}}\right)
$$

where, $\sigma$ controls the width of the Gaussian function. The large value of $\sigma$ gives a "fat bell-shape". However, the small value of $\sigma$ gives a "thin bell-shape". The $\boldsymbol{\beta}_{j}$ can be found by using the (11).

$$
\boldsymbol{\beta}_{j r}=G_{j}^{-1} y_{j r}
$$

The $\mathbf{G}_{j}$ may not be a square matrix as its inverse can be found by using the Moore-Penrose pseudo-inverse as shown in (12),

$$
\boldsymbol{\beta}_{j r}=\left(G_{j}^{-1} G_{j}\right)^{-1} G_{j} y_{j r}
$$

The structure of proposed FAM-RBF is demonstrated in Figure 1. As for training, the dataset is divided into training dataset and testing dataset to train and test this NN. In FAM-RBF, FAM is used to assist and compute the hidden neurons of RBF, and RBF is used in testing. Firstly, the training phase takes place with the presented training dataset. Then, the testing phase takes place with the presented testing dataset to test the accuracy of the FAM-RBF that constructed. When a training dataset that is newly presented to FAM$\mathrm{RBF}, \mathrm{FAM}$ is computed based on the (1) to (8). The hidden neurons of FAM together with their respective weights and centres, are presented to RBF after the training of FAM. A notable arrangement in FAM-RBF is the hidden neurons of FAM determine the centre of RBF hidden neurons. The hidden neurons of RBF are generated with (11). There are few user-defined parameters in FAM-RBF. Therefore, the process to determine the parameters has to be explored carefully to get a substantial performance results. After computing FAM-RBF, the consistency and persistence of the FAM-RBF have to be determined. The training and testing dataset are presented to the computed RBF of FAM-RBF to measure its training and testing accuracies [15].

\section{RESULTS AND ANALYSIS}

In this section, the performance of FAM-RBF on Harmonic Current Dataset is reported and compared with other popular NNs. The $\rho_{a}$ is set to 1 , and $\rho_{b}$ has to be tuned manually in this classification experiment. All the experiments in this paper were performed in an environment that listed in Table 1.

Table 1. Experiment Environment

\begin{tabular}{ll}
\hline Component & Specification \\
\hline Personal computer & HP Pavilion 14 Notebook PC \\
Operating system & Window 8.1 pro \\
RAM & 4.00 GB \\
Processor & Intel Core i5-3230M @ 2.6 GHz \\
Software & Matlab 7.11 (R2010b) \\
\hline
\end{tabular}

The objective of this paper is to classify types of consumers in Malaysia based on the Harmonic Currents Data provided (hereafter denote as Harmonic Current Dataset). There are total of 6936 samples in this dataset. The experiment in this paper had run for 50 trials to obtain average results. This strategy is needed as the results of FAM-RBF maybe impacted by random order of training sample. Each data sample 
has nine input and one output attributes. The input attributes are labelled from order-1 to order-9. Besides, the corresponding output, type of consumers, is classified from class 1 to class 5 respectively. The details of the class number and number of samples are listed in Table 2.

Table 2. Details Information of Harmonic Current dataset

\begin{tabular}{lcc}
\hline Types of consumers & Class number & Number of samples \\
\hline Residential Apartment & 1 & 939 \\
Hotel & 2 & 2010 \\
Bank & 3 & 1035 \\
Hospital & 4 & 2007 \\
Furniture Factory & 5 & 945 \\
\hline
\end{tabular}

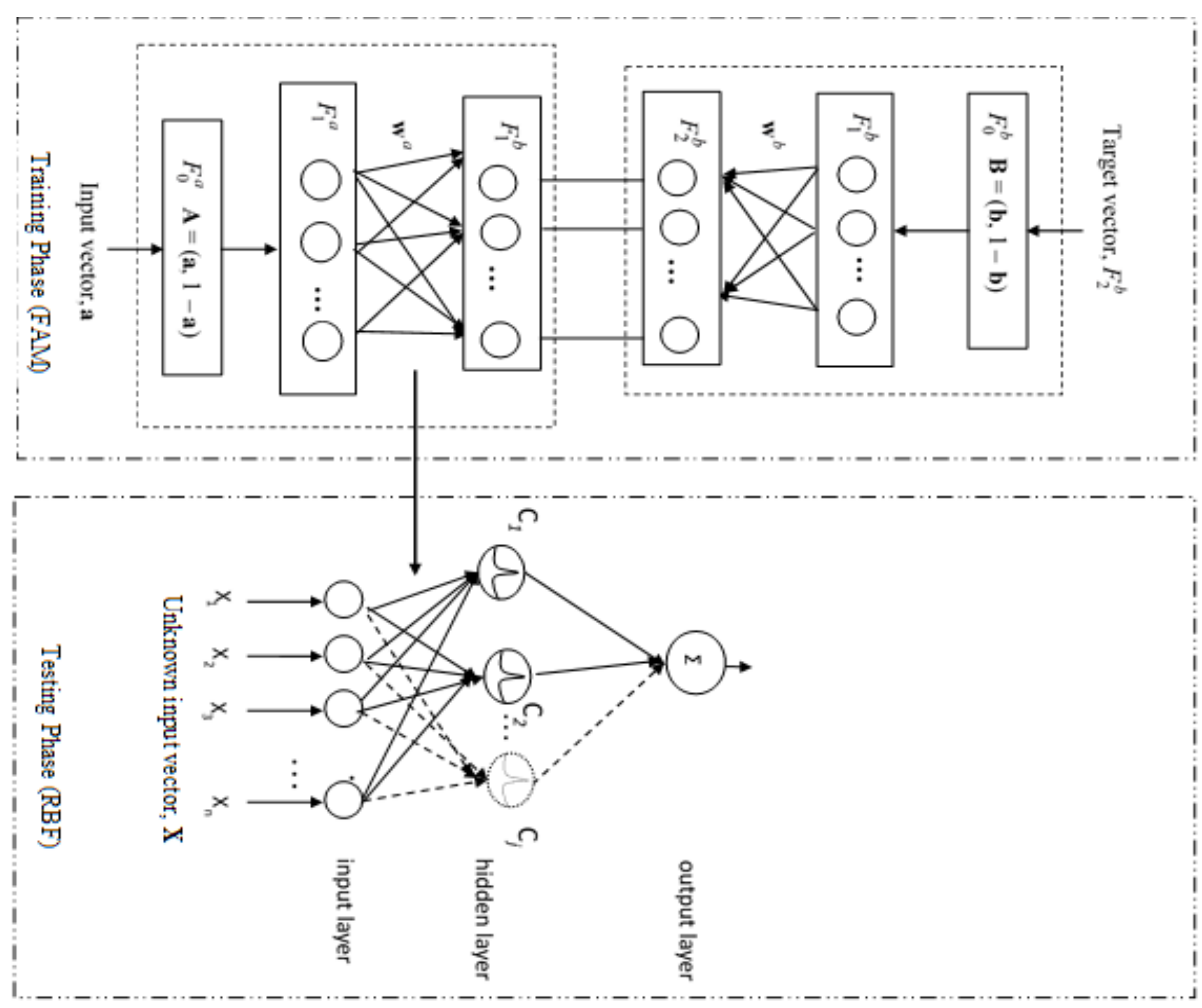

Figure 1. The Fuzzy ARTMAP- Radial Basis Function (FAM-RBF)

Table 3 presents the average training and testing accuracies of FAM-RBF and other popular NNs. The compared NNs are Improved Generalized Adaptive Resonance (IGART), Fuzzy ARTMAP (FAM), and Support Vector Machine (SVM) [3]. The IGART is a hybrid NN, which uses Gaussian ART to train and GRNN to test. It can be observed that the number of hidden neurons of FAM-RBF is significantly larger than all the compared NNs. Also, the testing accuracy of FAM-RBF (99.24\%) is larger than the compared NNs. The compared neural networks do not have training phase therefore there is no training accuracy reported. The training phase of FAM-RBF which is a phase to learn from the harmonic current classification, has improved its testing accuracy. This result has confirmed that the FAM-RBF is suitable to classify type of consumer based on harmonic current.

Table 3. Average Accuracy Rates for Harmonic Current Dataset

\begin{tabular}{cccc}
\hline Neural Network & \multicolumn{2}{c}{ Accuracy } & Number of hidden neurons \\
& Training & Testing & \\
\hline FAM-RBF & 99.68 & 99.24 & 307 \\
IGART & N/A & 98.92 & 16 \\
FAM & N/A & 98.79 & 15 \\
SVM & N/A & 98.92 & 204 \\
\hline
\end{tabular}


The number of hidden neurons of IGART in Table 3 is noted after pruning. The testing accuracy of IGART is $0.32 \%$ lesser than the testing accuracy of the proposed FAM-RBF, yet the number of hidden neurons of IGART is significantly 291 more than the proposed neural network. In real-world application, a NN is expected to have small number of hidden neurons. Dataset which consists of noise or redundant data, are unwanted by the user. The hidden neurons which are generated by both unwanted data, have to be removed by the NN. A large NN with abundant of hidden neurons due to overtraining is not welcomed and recommended in the application of NN [24-25]. During the training phase of FAM-RBF, its hidden neurons are generated as required by the presented data. A lot of hidden neurons were generated because the structure of the hidden neurons is sensitive to the sequence of presentation of data. Overlapping can occur easily due to the sequence presentation of data, and cause the $\mathrm{NN}$ to be overfitting. A further work has to be carried out to solve this overlapping problem.

The proposed model does not have a good knowledge learning model to solve overfitting problem. Specifically, this dataset is a complicated multi-class dataset because it had experienced two normalization process before dataset is presented to the proposed neural network. As a result, the computation time and cost are increased. Also, the selection of hidden neurons become complicated and tough. The right selection of learning model is important in $\mathrm{NN}$ to ensure the $\mathrm{NN}$ recognize and learn all the features of dataset. It may cause the NN to be underfitting or overfitting [26]. The technique to select an exact learning model for a NN is an exciting topic to be further studied in this research.

A further study on pruning unused hidden neurons without falling the both training and testing accuracies should be carried out. This pruning technique is aimed to reduce the complexity and computation cost of the neural network without a drastically change on its both training and testing accuracies. This technique can be either used during or after the training phase of proposed neural network.

\section{CONCLUSION}

This paper has proposed and exemplified the proposed FAM-RBF neural network can classify the types of consumers based on the Harmonic Currents Dataset that consolidated in Malaysia. The FAM is used to train the proposed neural network and facilitate the generation of RBF hidden neurons. When the RBF neural network is built, it is used to predict outputs. Both promising training and testing accuracies of proposed FAM-RBF neural network has shown that it is capable to handle multi-class classification.

Its testing accuracies were compared with other neural networks. The testing accuracy which is higher than the compared neural networks. This neural network has contributed to its interpretability as a suitable neural network to classify type of consumer based on the generated harmonic current generate a high accuracy with its high accuracy. In addition, the number of hidden neurons of FAM-RBF neural network is significantly larger than the compared neural networks. Some further studies can be done to strike a balance between accuracy and interpretability of the proposed neural network to solve this problem. The way to reduce the number of hidden neurons, complexity and computation time, and improve the performance of FAM-RBF should be further studied. Besides, the proposed neural network should be applied in other regression and multi-class classification cases to test its interpretability.

\section{ACKNOWLEDGEMENTS}

This work was supported by Universiti Tenaga Nasional (J510050684) and Xiamen University Malaysia (IECE/0001).

\section{REFERENCES}

[1] G. d. Castro, A. M. Muñoz and V. P. López, "Harmonic effect in street lighting," in 7th International Conference Workshop, Tallinn Estonia, 2011.

[2] J. Caicedo, A. R. d. Castro, B. França and a. M. Aredes, "Resonant harmonic compensation for synchronverter, integrating wind and photovoltaic power generation into an electrical grid, case study: Nonlinear and unbalanced load," in Power Electronics Conference (COBEP), 2017 Brazilian, Juiz de Fora, Brazil, 2017.

[3] K. S. Yap, C. P. Lim and M. T. Au, "Improved GART Neural Network Model for Pattern Classification and Rule Extraction with Application to Power Systems ," IEEE Transactions on Neural Networks, vol. 22, no. 12, pp. $2310-2323,2011$.

[4] P. W. J. M. D. F. a. Y.-A. C. Djaffar Ould Abdeslam, “A Unified Artificial Neural Network Architecture for Active Power Filters, ” IEEE Transactions on Industrial Electronics, vol. 54, no. 1, pp. 61-76, 2007.

[5] K. Metaxiotis, A. Kagiannas, D. Askounis and J. Psarras, "Artificial intelligence in short term electric load forecasting: a state-of-the-art survey for the researcher," Energy Conversion and Management, vol. 44, no. 9, pp. 1525-1534, 2003. 
[6] M. A. Mat Daut, M. Y. Hassan, H. Abdullah, H. Abdul Rahman, M. P. Abdullah and F. Hussin, "Building electrical energy consumption forecasting analysis using conventional and artificial intelligence methods: A review," Renewable and Sustainable Energy Reviews, vol. 70, pp. 1108-1118, 2017.

[7] G. W. Chang, H. J. Lu, Y. Y. Chen and Y. R. Chang, "Forecasting wind power generation by a new type of radial basis function-based neural network," in 2017 IEEE Power \& Energy Society General Meeting, Chicago, IL, USA, 2017.

[8] H. Zareipour, D. Huang and W. Rosehart, "Wind power ramp events classification and forecasting: A data mining approach," in 2011 IEEE Power and Energy Society General Meeting, San Diego, CA, USA, 2011.

[9] G. A. Carpenter, S. Grossberg, N. Markuzon and J. H. Reynolds, "Fuzzy ARTMAP: A neural network architecture for incremental supervised learning of analog multidimensional maps," IEEE Trans. Neural Netw., vol. 3, no. 5, pp. 698-713, 1992.

[10] G. A. Carpenter, G. G. S. and a. R. J. H., “ARTMAP: Supervised real-timelearning and classification of monstationary data by a self-organizing neural network," Neural Networks, vol. 4, p. 565-588, 1991.

[11] G. Carpenter and S. Grossberg, "A self-organizing neural network for supervised learning, recognition, and prediction," IEEE Communications Magazine, vol. 30, no. 9, pp. 38-49, September 1992.

[12] De and N. Chatterjee, "A Fuzzy ARTMAP Fault Classifier for Impulse Testing of Power Transformers," IEEE Transactions on Dielectrics and Electrical Insulation, vol. 11, no. 6, pp. 1026-1036, 2004.

[13] W. Y. Sit, L. O. Mak and G. W. Ng, "Managing Category Proliferation in Fuzzy ARTMAP Caused by Overlapping Classes," IEEE Transactions on Neural Networks, vol. 20, no. 8, pp. 1244-1253, 2009.

[14] S. Tan and C. P. Lim, "Evolutionary Fuzzy ARTMAP Neural Networks and Their Applications to Fault Detection and Diagnosis," Neural Processing Letters, vol. 31, p. 219-242, 2010.

[15] S. Y. Leow, S. Y. Wong, K. S. Yap and H. J. Yap, "A new hybrid fuzzy ARTMAP and radial basis function neural network with online pruning strategy," in 2016 7th IEEE Control and System Graduate Research Colloquium (ICSGRC), Shah Alam, Malaysia, 2016.

[16] D. Charalampidis, T. Kasparis and M. Georgiopoulos, "Classification of Noisy Signals Using Fuzzy ARTMAP Neural Networks,” IEEE Transactions on Neural Networks, vol. 12, no. 5, pp. 1023-1036, 2001.

[17] Z.-R. Lai, D.-Q. Dai, C.-X. Ren and K.-K. Huang, "Radial Basis Functions With Adaptive Input and Composite Trend Representation for Portfolio Selection," IEEE Transactions on Neural Networks and Learning Systems, pp. 1-13, 2018.

[18] D. G. Barros Franco and M. T. Arns Steiner, "New Strategies for Initialization and Training of Radial Basis Function Neural Networks, ” IEEE Latin America Transactions, vol. 15, no. 6, pp. 1182-1188, 2017.

[19] H. R. Baghaee, M. Mirsalim, G. B. Gharehpetian and A. T. Heidar, "Unbalanced harmonic power sharing and voltage compensation of microgrids using radial basis function neural network-based harmonic power-flow calculations for distributed and decentralised control structures," IET Generation, Transmission \& Distribution, vol. 12, no. 7, pp. 1518-1530, 2018.

[20] J. Morel, A. Bac and C. Véga, "Terrain Model Reconstruction from Terrestrial LiDAR Data Using Radial Basis Functions," IEEE Computer Graphics and Applications, vol. 37, no. 5, pp. 72-84, 2017.

[21] Y. Li, X.-D. Wang, M.-L. Luo, K. Li, X.-F. Yang and Q. Guo, "Epileptic Seizure Classification of EEGs Using Time-Frequency Analysis Based Multiscale Radial Basis Functions," IEEE Journal of Biomedical and Health Informatics, vol. 22, no. 2, pp. 386-397, 2018.

[22] K. M. Tay and C. P. Lim, "On the Use of Fuzzy Inference Techniques in Assessment Models: Part II: Industrial Applications," Fuzzy Optimization and Decision Making, vol. 7, no. 3, pp. 283-302, 2008.

[23] J. Park and I. W. Sandberg, "Universal Approximation using Radial-Basis-Function Networks," Neural Computation, vol. 3, pp. 246-2S7, 1991.

[24] 1. Y. Alanis, "Electricity Prices Forecasting using Artificial Neural Networks," IEEE Latin America Transactions, vol. 16, no. 1, pp. 105-111, 2018.

[25] D. Marček and M. Rojček, "The category proliferation problem in ART neural networks," Acta Polytechnica Hungarica, vol. 14, no. 5, pp. 49-63, 2017.

[26] F. S. Panchal and M. Panchal, "Review on Methods of Selecting Number of Hidden Nodes in Artificial Neural Network," International Journal of Computer Science and Mobile Computing, vol. 3, no. 11, p. 455-464, 2014.

\section{BIOGRAPHIES OF AUTHORS}

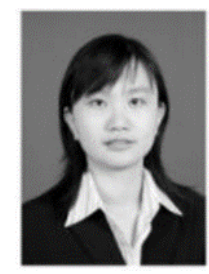

Shoun Ying Leow received the Bachelor's degree with Honors in Electrical and Electronics Engineering, Master and Ph.D. in Engineering degrees in Electrical Engineering from Universiti Tenaga Nasional (UNITEN), Malaysia in 2011, 2013 and 2018. Prior her PhD study, she worked as a lecturer. Her research interests include artificial neural network, machine learning and pattern recognition. 

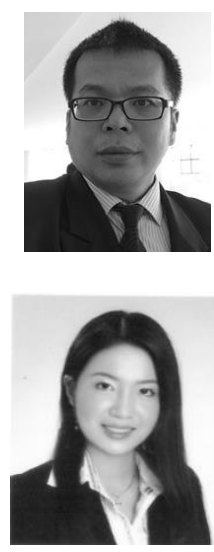

Keem Siah Yap received his B. Eng (Electrical) (Hons.) and M.Sc (Electrical Engineering) degree both from Universiti Teknologi Malaysia in 1998 and 2000 respectively. In year 2010, he received PhD in Electronics Engineering from Universiti Sains Malaysia. His research interests include theory and applications of artificial intelligence. Currently, he is a Professor at College of Engineering, Universiti Tenaga Nasional, Malaysia.

Shen Yuong Wong received her Bachelor Degree and M.Sc. of Electrical and Electronic Engineering with first class honors from Universiti Tenaga Nasional, Malaysia in 2010, and 2012 respectively. She received her $\mathrm{PhD}$ degree in Engineering from the same university in 2015. Her research interests include application of artificial intelligence, pattern recognition, fuzzy logic and Extreme Learning Machines. Currently she is an Assistant Professor at Department of Electrical and Electronics Engineering, Xiamen University Malaysia, Malaysia. 